\title{
Resveratrol Pretreatment Ameliorates p53-Bax Axis and Augments the Survival Biomarker B-Cell Lymphoma 2 Modulated by Paracetamol Overdose in a Rat Model of Acute Liver Injury
}

\author{
Suliman Al Humayed ${ }^{a}$ Fahaid Al-Hashem $^{b}$ Mohamed A. Haidara ${ }^{b} c$ \\ Abbas O. El Karib ${ }^{b}$ Samaa S. Kamar ${ }^{d}$ Shaimaa N. Amin ${ }^{c}$ Bahjat Al-Ani ${ }^{\text {b }}$ \\ ${ }^{a}$ Department of Internal Medicine, College of Medicine, King Khalid University, Abha, Saudi Arabia; ${ }^{b}$ Department

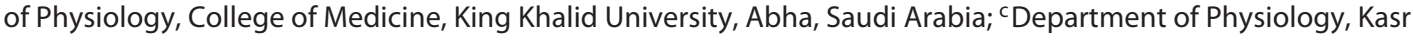 \\ Al-Aini Faculty of Medicine, Cairo University, Cairo, Egypt; ${ }^{d}$ Department of Medical Histology, Kasr Al-Aini Faculty of \\ Medicine, Cairo University, Cairo, Egypt
}

\section{Keywords}

Acute liver injury · p53-Bax axis · Paracetamol · Resveratrol · B-cell lymphoma $2 \cdot$ Rat model

\begin{abstract}
Background: The potential protective effects of resveratrol (RES) on the modulation of hepatic biomarkers of apoptosis and survival, p53-Bax axis, and B-cell lymphoma 2 (Bcl-2) in an animal model of paracetamol-induced acute liver injury have not been investigated before. Methods: The model group of rats received a single dose of paracetamol $(2 \mathrm{~g} / \mathrm{kg}$, orally), whereas the protective group of rats were pretreated for 7 days with RES (30 mg/kg, i.p.) before they were given a single dose of paracetamol. All rats were then sacrificed 24-h post paracetamol ingestion. Results: Histology images showed that paracetamol overdose induced acute liver injury, which was substantially protected by RES. Paracetamol significantly $(p<0.05)$ modulated $\mathrm{p} 53$, apoptosis regulator $\mathrm{Bax}, \mathrm{Bcl}-2$, tumor necrosis factor-alpha, interleukin-6, inducible nitric oxide synthase, malondialdehyde, superoxide dismutase, glutathione peroxidase, alanine aminotransferase,
\end{abstract}

and aspartate aminotransferase, which were significantly protected by RES. We further demonstrated a significant $(p<$ 0.01 ) correlation between either $\mathrm{p} 53$ or $\mathrm{Bcl}-2$ scoring and the levels of inflammatory, nitrosative stress, and liver injury biomarkers. Conclusion: We demonstrate a substantial protection by RES pretreatment against paracetamol-induced modulation of $\mathrm{p} 53-\mathrm{Bax}$ axis, $\mathrm{BCl}-2$, and other acute liver injury biomarkers in rats.

(c) 2019 S. Karger AG, Basel

\section{Introduction}

Paracetamol (also called acetaminophen [APAP]) inducing hepatotoxicity is common in both humans and experimental animal models after excessive use or ingestion of an overdose of the drug [1]. It is the most common agent of intentional self-harm, and APAP poisoning claimed the life of 284 persons aged 12 years and over between 1993 and 1996 in England and Wales, UK [2]. In addition, about $50 \%$ of acute liver failure admitted cases in the United States of America are caused by APAP poi-

\section{KARGER}

(c) 2019 S. Karger AG, Basel

E-Mail karger@karger.com

www.karger.com/pha
Prof. Bahjat Al-Ani

Department of Physiology

College of Medicine, King Khalid University

Abha 61421 (Saudi Arabia)

E-Mail bahjat_alani@yahoo.com 
soning $[3,4]$. APAP is metabolized in the liver, and hepatotoxic metabolites that represent about $10 \%$ of the whole metabolites are rapidly inactivated by glutathione (GSH) to protect the hepatocytes [5]. But, with the drug overdose, for example, the elevated levels of liver toxic metabolites, mainly $\mathrm{N}$-acetyl-p-benzoquinoimine rapidly depletes GSH and covalently modifies cellular proteins. This leads to the generation of high levels of reactive oxygen species (ROS) and depletion of the ATP, which results in mitochondrial damage and hepatocyte injury [6]. It was postulated that depletion of $90 \%$ of GSH in hepatocytes is critically necessary for the development of cell necrosis [7]. In addition, hepatic inflammatory cytokines are also reported to be involved in APAP-induced liver injury [8].

Resveratrol (RES) is a polyphenolic anti-inflammatory and antioxidant found in grapes, berries, and peanuts [9]. It has been widely known to have potent cardiovascular protective and therapeutic effect via scavenging ROS [10], anti-inflammatory effects [11], inhibit lipid peroxidation [12], inhibit platelet aggregation and thrombus formation [13], and liver protection against hepatic sinusoidal obstruction and hepatic steatosis [14]. In addition, RES given immediately after APAP injection was reported to prevent oxidative damage in hepatic tissues in mice $(4 \mathrm{~h})$ post APAP poisoning [15]. Thus, it is likely that RES given daily for 7 days before APAP ingestion can protect the modulation of biomarkers of acute liver injury such as hepatic gene expression of p53-Bax axis and B-cell lymphoma 2 (Bcl-2) protein in rats.

\section{Methods}

\section{Experimental Design}

We followed the regulations of animal use published by the US National Institutes of Health (publication No. 85-23, revised 1996). Male Wistar rats (10 weeks; $180-200$ g) were used in this study, and after a 1 week adaptation period, rats were randomly assigned into 4 groups ( $n=6$; each) and were distributed in their corresponding cages and classified as follows: (1) Control group: rats received normal saline i.p. daily for 7 days; (2) RES group: rats were pretreated with RES (30 mg/kg, i.p.) [15] for 7 consecutive days; (3) APAP intoxicated group (Model group): rats received normal saline for 7 consecutive days and then were given a single dose of APAP (2 g/kg, orally) [16]; (4) RES + APAP group: rats were pretreated with RES (30 mg/kg) [15] for 7 consecutive days and then were administered with a single dose of APAP $(2 \mathrm{~g} / \mathrm{kg}$, orally). APAP was administered to the desired groups $1 \mathrm{~h}$ after the last dose of treatment administration on day 7. At the end of experimental period, 24 -h post APAP treatment, blood samples were collected by cardiac puncture under anaesthesia (sodium thiopental at $40 \mathrm{mg} / \mathrm{kg}$ body weight), and animals were then culled. Harvested liver tissues were prepared for histological staining, and liver homogenates were prepared as we previously reported [17].

\section{Histological Study}

Liver specimens were dissected out by midline laparotomy and immediately fixed in $10 \%$ formol saline for $24 \mathrm{~h}$. Paraffin blocks were processed, sectioned in $5 \mu \mathrm{m}$ thickness, and subjected to $\mathrm{H} \& \mathrm{E}$ staining to observe the morphological changes [18].

\section{Detection of 553 and Bax Messenger RNAs by Reverse}

Transcriptase-Polymerase Chain Reaction

Total RNAs were isolated from freshly dissected rats' livers using the RNeasy Mini Kit (Qiagen Pty, Victoria, Australia). The RNA was reverse-transcribed for a single-strand cDNA synthesis (Invitrogen) and amplified by polymerase chain reaction (PCR) using primer pairs for $\mathrm{p} 53, \mathrm{Bax}$, and $\beta$-actin derived from the published cDNA sequence as previously described by our group [19]. The PCR products were separated by $2 \%$ agarose gel electrophoresis and visualized by ethidium bromide, and gel images were scanned and quantified by densitometry using the National Institutes of Health image software.

Determination of Blood and Tissue Levels of Alanine

Aminotransferase, Aspartate Aminotransferase, Bcl-2,

Inducible Nitric Oxide Synthase, Malondialdehyde, Superoxide

Dismutase, GSH Peroxidase, Tumor Necrosis Factor-Alpha,

and Interleukin-6

Assay kits for determination of malondialdehyde (MDA) were purchased from NWLSS (Vancouver, BC, Canada). Superoxide dismutase (SOD) and GSH peroxidase (GPx) activities were measured using a commercial assay kits (Cayman Chemical, Ann Arbor, MI, USA). Assay kits for determination of serum levels of alanine aminotransferase (ALT) and aspartate aminotransferase (AST) were purchased from Human Co., Germany. Enzyme-linked immunosorbent assay (ELISA) kits for determination of tumor necrosis factor-alpha (TNF- $\alpha$ ) were purchased from Abcam, Cambridge, UK. ELISA kits for determination of the levels of interleukin-6 were purchased from RayBio, GA, USA. ELISA kits for determination of the levels of BCl-2 and inducible nitric oxide synthase (iNOS) were purchased from STZ ELISA, USA.

\section{Statistical Analysis}

Statistical analyses were performed by using GraphPad prism statistical software package (version 6). Data were presented as means with their SD (mean \pm SD). Normality and homogeneity of the data were confirmed before ANOVA, differences among the experimental groups were assessed by one-way ANOVA followed by Tukey's $t$ test.

\section{Results}

\section{RES Reduces APAP-Induced Biomarkers of Liver}

Injury Enzymes, ALT and AST

APAP-induced hepatotoxicity caused about a 5-fold increase in ALT and a 7-fold increase in AST, compared to the control groups (Table 1). Pretreatment with RES significantly inhibited ALT $(p \leq 0.007)$ and AST $(p<$ 0.0001).
Al Humayed/Al-Hashem/Haidara/

El Karib/Kamar/Amin/Al-Ani 
Table 1. Effect of RES on paracetamol (APAP)-induced liver injury enzymes

\begin{tabular}{lllll}
\hline Liver injury enzymes & Control group & RES group & APAP group & RES + APAP group \\
\hline ALT, U/mL & $27.12 \pm 3.43$ & $27.03 \pm 3.40$ & $121.3 \pm 17.69^{*}$ & $56.32 \pm 10.58^{*}$, \# \\
AST, U/L & $24.50 \pm 3.59$ & $24.23 \pm 3.51$ & $166.0 \pm 12.98^{*}$ & $39.58 \pm 3.93^{*}$, \# \\
\hline
\end{tabular}

Blood levels of ALT and AST were measured in all experimental groups of rats. Values are expressed as mean \pm SD for 6 rats in each group. Values were considered significantly different at $p<0.05$.

* Significant in comparison to control.

\# Significant in comparison to APAP.

RES, resveratrol; APAP, acetaminophen; ALT, alanine aminotransferase; AST, aspartate aminotransferase.

Fig. 1. RES protects the modulation of proapoptotic and antiapoptotic biomarkers caused by APAP. Liver tissue levels of p53 and Bax gene expression were measured using RT-PCR analysis (a-c), and Bcl-2 protein (d) measured by ELISA; Control (lane 1 for a), RES (lane 2 for a), APAP (lane 3 for a), and RES + APAP (lane 4 for a) groups. Results represent the mean $( \pm \mathrm{SD}) ; n=6$ for each group. Experiments were performed in triplicate. All shown $p$ values are significant. ${ }^{*} p<0.0001$ versus control, $\# p \leq 0.03$ versus APAP. mRNA, messenger mRNA; RES, resveratrol; APAP, acetaminophen; Bcl-2, B-cell lymphoma 2.

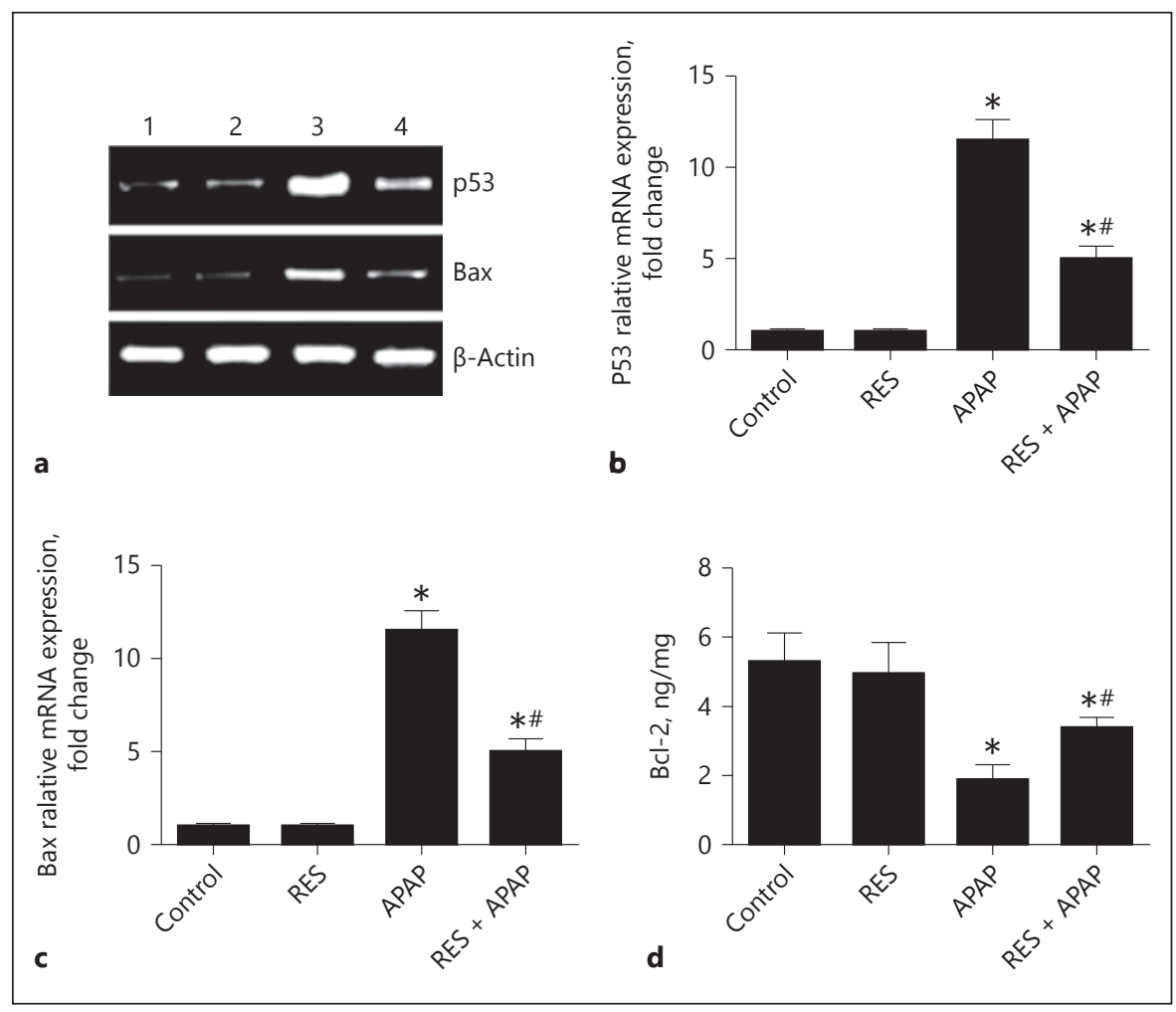

\section{RES Protects APAP Modulated Apoptosis and}

\section{Survival Biomarkers in Liver Tissues}

We investigated in a rat model of acute liver injury induced by APAP whether RES can modulate the apoptotic biomarker Bax and the antiapoptotic protein $\mathrm{Bcl}-2$ that are located downstream of p53 in hepatocyte mitochondrial damage [20]. Thus, we assessed the expression of messenger RNAs for p53 and Bax genes using reverse transcriptase-PCR and also measured the levels of the survival protein Bcl-2 by ELISA. Acute liver injury induced by APAP-augmented p53 ( 13 fold) and Bax ( 15 fold) messenger RNA messages (Fig. 1b, c), and ameliorated Bcl-2
(3 fold; Fig. 1d). Pretreatment with RES substantially inhibited p53 (about $60 \%$ reduction; Fig. 1b) and Bax (about $70 \%$ reduction; Fig. 1c) gene expression, and significantly $(p<0.0001)$ augmented Bcl-2 levels (Fig. 1d).

\section{RES Protects the Modulation of Nitrosative and Oxidative Stresses and Antioxidants Biomarkers Induced by APAP in Liver Tissues}

To investigate the level of inhibition of biomarkers of oxidative and nitrosative stresses by RES using our protective approach, we measured MDA as a by-product of lipid peroxidation and iNOS in liver homogenates of all groups. 
Fig. 2. RES protects the modulation of nitrosative and oxidative stress, and antioxidant biomarkers caused by APAP. Liver tissue levels of iNOS (a), MDA (b), SOD (c), and GPx (d) enzymes were measured in all groups of rats; Control, RES, APAP, and RES + APAP groups. Results represent the mean $( \pm \mathrm{SD})$; $n=6$ for each group. Experiments were performed in triplicate. All shown $p$ values are significant. ${ }^{*} p \leq 0.0009$ versus control, $\# p \leq 0.03$ versus APAP. iNOS, inducible nitric oxide synthase; RES, resveratrol; APAP, acetaminophen; MDA, malondialdehyde; SOD, superoxide dismutase; GPx, glutathione peroxidase.

Fig. 3. RES protects against APAP-induced inflammation. Liver tissue levels of TNF- $\alpha$ (a) and IL-6 (b) were measured in all groups of rats; Control, RES, APAP, and RES + APAP groups. Results represent the mean $( \pm \mathrm{SD}) ; n=6$ for each group. Experiments were performed in triplicate. All shown $p$ values are significant. ${ }^{*} p \leq 0.02$ versus control, ${ }^{\#} p \leq 0.007$ versus APAP. TNF- $\alpha$, tumor necrosis factor-alpha; RES, resveratrol; APAP, acetaminophen; IL-6, interleukin-6.
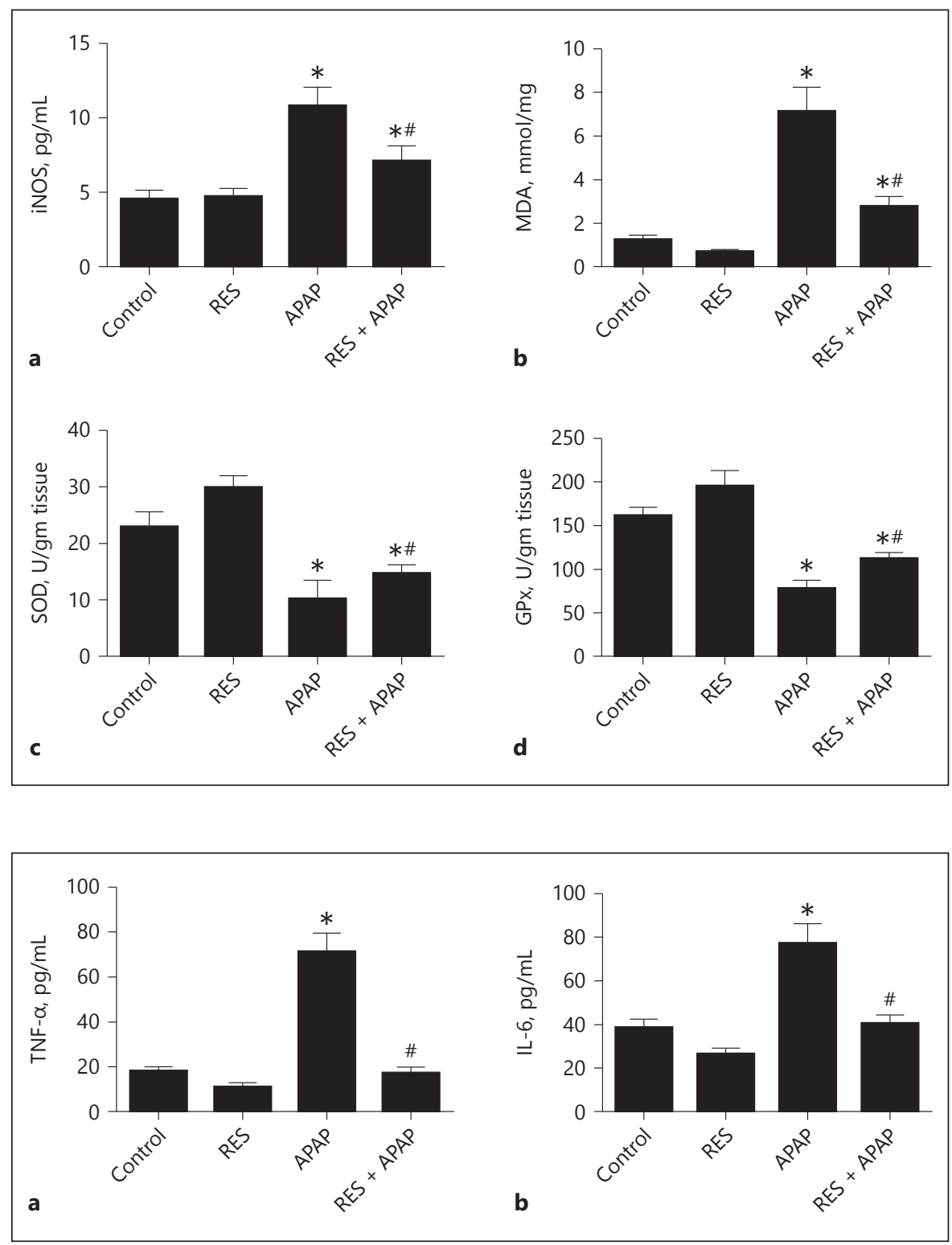

As shown in Figure $2 \mathrm{a}$ and $\mathrm{b}$, acute liver injury significantly $(p<0.0001)$ augmented iNOS and MDA levels compared to the control groups that were significantly inhibited by RES $(p<0.0001)$ compared to the model group.

We further measured liver tissue levels of the antioxidants, SOD (Fig. 2c) and GPx (Fig. 2d) in all rat groups. APAP inhibited both SOD and GPx, which were augmented by RES.

\section{RES Reduces APAP-Induced Biomarkers of \\ Inflammation}

The level of inhibition of inflammation in liver homogenates upon pretreatment of rats with RES for 7 days was investigated. Compared to the model group, a significant inhibition of TNF- $\alpha$ and interleukin-6 ( $p<$ 0.0001; Fig. 3a, b) by RES were observed that brought blood levels of these biomarkers to levels comparable to controls.

\section{Correlation between p53 or Bcl-2 and Biomarkers of Inflammation, Nitrosative Stress, and Liver Injury \\ Enzyme \\ We determined the correlation between either p53 or} Bcl-2 scorning and the tissue levels of inflammation and nitrosative stress and serum levels of liver injury biomarkers in order to further confirm and characterize that
42

Pharmacology 2020;105:39-46 DOI: $10.1159 / 000502632$
Al Humayed/Al-Hashem/Haidara/ El Karib/Kamar/Amin/Al-Ani 


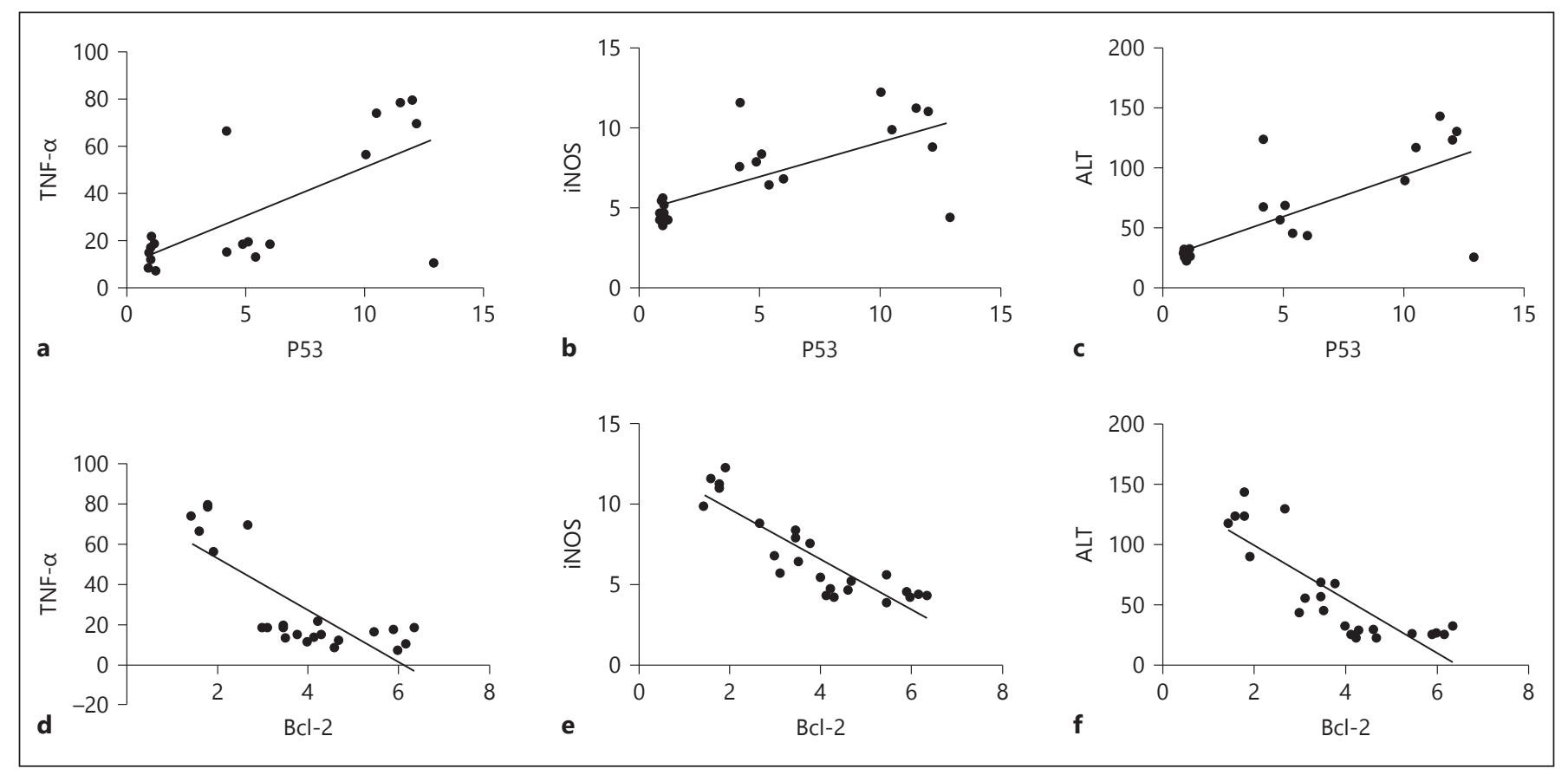

Fig. 4. Correlation between p53 or Bcl-2 and biomarkers of inflammation, nitrosative stress, and liver injury enzyme. P53 and Bcl-2 liver homogenate levels were measured in all groups of rats and the positive correlation between p53 and TNF- $\alpha$, p53 and iNOS, and p53 and ALT are shown in (a-c), respectively. The negative cor-

the role of RES is stable and an appropriate agent in liver injury rats and to further support the link between p53 and $\mathrm{Bcl}-2$ and liver injury. Figure $4 \mathrm{a}-\mathrm{c}$ show a positive correlation between p53 and these biomarkers; p53 versus TNF- $\alpha(r=0.722 ; p=0.0001)$, p53 versus iNOS $(r=$ $0.695 ; p=0.0002)$, and p53 versus ALT ( $r=0.747 ; p<$ $0.0001)$. Whereas, a negative correlation was shown between Bcl-2 and TNF- $\alpha(r=-0.786 ; p<0.0001$; Fig. $4 d)$, Bcl-2 versus iNOS ( $r=-0.878 ; p<0.0001$; Fig. $4 \mathrm{e})$, and Bcl-2 versus ALT ( $r=-0.844 ; p<0.0001$; Fig. $4 \mathrm{f})$.

\section{RES Partially Protects Liver Tissue against}

\section{APAP-Induced Injury}

H\&E stained sections of liver from the model group (APAP) showed evidence of substantial damage to the hepatocytes, which is demonstrated by multiple necrotic foci with infiltrating inflammatory cells, hemorrhage, and congestion of blood vessels (Fig. 5c) compared with a normal architecture of the liver parenchyma in the control groups displaying cords of hepatocytes radiating from central vein and surrounding portal tract (Fig. 5a, b). RES treatment preserved liver architecture (Fig. 5d). However, few inflammatory cell infiltrations were still seen. relation between Bcl-2 and TNF- $\alpha$, Bcl-2 and iNOS, and Bcl-2 and ALT are shown in (d-f), respectively. TNF- $\alpha$, tumor necrosis factor-alpha; Bcl-2, B-cell lymphoma 2; iNOS, inducible nitric oxide synthase; ALT, alanine aminotransferase.

\section{Discussion}

In this report, we induced acute liver injury in rats, which developed 24 -h post APAP ingestion. We were required to generate this animal model in order to investigate the effects of RES on (i) APAP-induced the proapoptotic p53-Bax gene expression in liver and (ii) APAP-inhibited the survival (antiapoptotic) protein Bcl-2 in liver tissue homogenates. In addition, we addressed the level of protection provided by RES to liver tissue architecture and the modulation of selected blood and tissue biomarkers such as oxidative and nitrosative stress, inflammation, and liver injury enzymes, which occurred upon the induction of acute liver injury in rats. Depletion of GSH and upregulation of ROS by APAP due to N-acetyl-p-benzoquinoimine accumulation cause liver injury (5-8) that involves leukocyte infiltration, inflammation, iNOS upregulation, apoptosis, and necrosis [21, 22]. Therefore, our investigation was justified since the antioxidant compound RES is known to treat liver injury by increasing hepatic GSH in APAP- and CCl4-induced acute liver injuries $[15,23]$. In addition, RES was reported to alleviate the deleterious effects of other hepatotoxic agents such as 


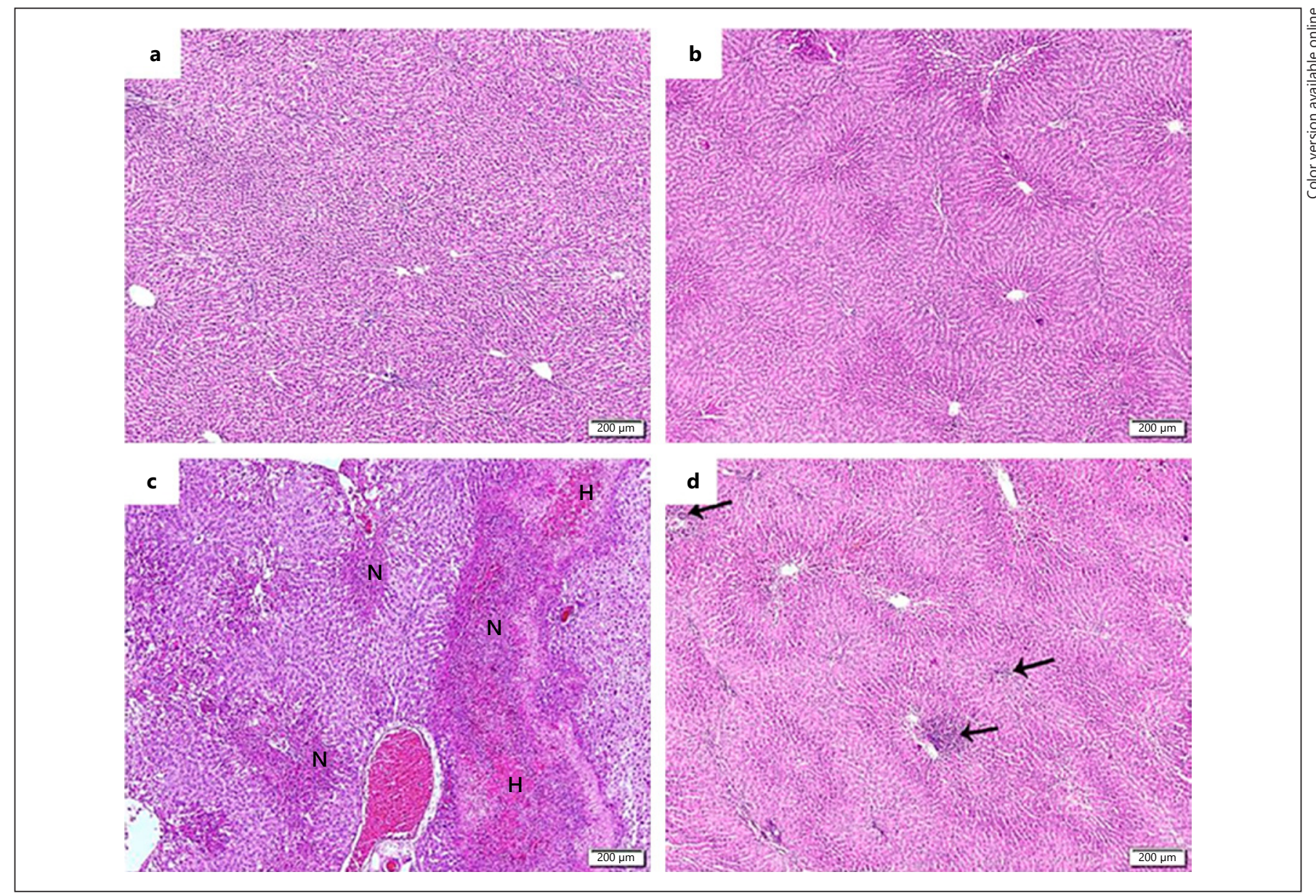

Fig. 5. RES partially protects liver architecture against liver injury induced by APAP. H\&E stained images $(\times 40)$ of harvested tissues obtained from the liver of the control group (a), RES group (b),
APAP group (c), and the protective group, RES + APAP (d). Note that arrows in (d) point to inflammatory cell infiltration. $\mathrm{N}$, multiple necrotic foci; $\mathrm{H}$, hemorrhage. paraquat via the inhibition of oxidative and nitrosative stress, inflammation, and apoptosis [24].

This study is the first to report that RES inhibits the modulation of hepatic biomarkers of apoptosis and survival, p53-Bax axis, and Bcl-2 in an animal model of APAP-induced acute liver injury. Our data that showed APAP overdose inhibited the antiapoptotic protein Bcl-2 (Fig. 1d), which is in agreement with previous work that showed APAP induce caspase-dependent apoptosis, and overexpression of the antiapoptotic protein $\mathrm{Bcl} 3$ in human hepatoma cells and lymphocytes inhibited apoptosis [25]. In addition, our data are also in agreement with at least 2 studies [24, 26], which reported an inhibition of Bcl-2 levels in a rat model of neonatal brain injury after hypoxia-ischemia and a mouse model of paraquat-induced hepatic injury, and RES treatment significantly augmented Bcl-2 levels. In addition, our data that point to the upregulation of iNOS in liver homogenates by APAP and its significant inhibition with RES (Fig. 2a) are in agreement and complement the recently published work [27] that reported a significant inhibition of liver iNOS immunostaining by RES given 20 -min post APAP injection in rats. Furthermore, correlation between apoptosis or antiapoptosis and liver injury biomarkers is shown in Figure 4, which clearly links these parameters, is in agreement with previous work that dissected the signal transduction of tissue injuries via inflammation, oxidative stress, and apoptosis pathways [28].

Treating mice with RES prior to the induction of hepatotoxicity by APAP negatively regulated the apoptotic protein p53 and positively regulated the survival protein SIRT1 cell signaling to promote hepatocyte proliferation [22], which is in agreement with the p53 part of our data and further supports our work that demonstrated an up- 
regulation of the survival protein $\mathrm{Bcl}-2$ and downregulation of the apoptosis regulator Bax gene expression by RES. Also, our data that point to the inhibition of APAPinduced p53, Bax, MDA, TNF- $\alpha$, ALT, and AST by RES are in agreement with recently published work on RES inhibition of these biomarkers in a mouse model of hepatic injury induced by paraquat [24]. Finally, RES administered at the same time with APAP to rats [29] caused no significant pathological changes in the liver tissue of the model group (APAP) stained with H\&E. Whereas, a representative of our H\&E images shown in Figure $5 c$ demonstrated substantial liver damage induced by APAP. Our treatment protocol $(2 \mathrm{~g} / \mathrm{kg}$ APAP) versus their treatment protocol ( $1 \mathrm{~g} / \mathrm{kg}$ APAP) could also be the reason of such differences.

Collectively, these studies support the conclusion that RES protects against APAP-induced liver tissue injury and modulation of the p53-Bax axis, Bcl-2, and hepatic biomarkers of acute liver injury such as augmentation of hepatic levels of antioxidants and inhibition of inflammation and oxidative stress in rats, 24 -h post APAP ingestion.

\section{Acknowledgments}

The author would like to thank Dr. Mariam Al-Ani from Dental Care Partnership, Sutton Coldfield, Birmingham, UK, for proofreading the manuscript.

\section{Disclosure Statement}

The authors declare that there are no conflicts of interest.

\section{Funding Sources}

King Khalid University, Grant/Award Number: KKU-Project No. R.G.P1./88/40.

\section{References}

1 McGill MR, Sharpe MR, Williams CD, Taha M, Curry SC, Jaeschke H. The mechanism underlying acetaminophen-induced hepatotoxicity in humans and mice involves mitochondrial damage and nuclear DNA fragmentation. J Clin Invest. 2012 Apr;122(4):1574-83.

2 Hawton K, Simkin S, Deeks J, Cooper J, Johnston A, Waters K, et al. UK legislation on analgesic packs: before and after study of long term effect on poisonings. BMJ. 2004 Nov; 329(7474):1076.

3 Ostapowicz G, Fontana RJ, Schiødt FV, Larson A, Davern TJ, Han SH, et al.; U.S. Acute Liver Failure Study Group. Results of a prospective study of acute liver failure at 17 tertiary care centers in the United States. Ann Intern Med. 2002 Dec;137(12):947-54.

4 Larson AM, Polson J, Fontana RJ, Davern TJ, Lalani E, Hynan LS, et al.; Acute Liver Failure Study Group. Acetaminophen-induced acute liver failure: results of a United States multicenter, prospective study. Hepatology. 2005 Dec;42(6):1364-72

5 James LP, Mayeux PR, Hinson JA. Acetaminophen-induced hepatotoxicity. Drug Metab Dispos. 2003 Dec;31(12):1499-506.

6 Hinson JA, Reid AB, McCullough SS, James LP. Acetaminophen-induced hepatotoxicity: role of metabolic activation, reactive oxygen/ nitrogen species, and mitochondrial permeability transition. Drug Metab Rev. 2004 Oct; 36(3-4):805-22.

7 Henderson CJ, Wolf CR, Kitteringham N, Powell H, Otto D, Park BK. Increased resistance to acetaminophen hepatotoxicity in mice lacking glutathione S-transferase Pi. Proc Natl Acad Sci USA. 2000 Nov;97(23): 12741-5.

8 Blazka ME, Elwell MR, Holladay SD, Wilson RE, Luster MI. Histopathology of acetaminophen-induced liver changes: role of interleukin 1 alpha and tumor necrosis factor alpha. Toxicol Pathol. 1996 Mar-Apr;24(2):181-9.

9 Cudmore MJ, Ramma W, Cai M, Fujisawa T, Ahmad S, Al-Ani B, et al. Resveratrol inhibits the release of soluble fms-like tyrosine kinase (sFlt-1) from human placenta. Am J Obstet Gynecol. 2012 Mar;206(3):253.e10-5.

10 Hung LM, Chen JK, Huang SS, Lee RS, Su MJ. Cardioprotective effect of resveratrol, a natural antioxidant derived from grapes. Cardiovasc Res. 2000 Aug;47(3):549-55.

11 Al-Ani B. Resveratrol inhibits proteinase-activated receptor-2-induced release of soluble vascular endothelial growth factor receptor-1 from human endothelial cells. EXCLI J. 2013 Jul;12:598-604.

12 Frankel EN, Waterhouse AL, Kinsella JE. Inhibition of human LDL oxidation by resveratrol. Lancet. 1993 Apr;341(8852):1103-4.

13 Bertelli AA, Giovannini L, Giannessi D, Migliori M, Bernini W, Fregoni M, et al. Antiplatelet activity of synthetic and natural resveratrol in red wine. Int J Tissue React. 1995; 17(1):1-3

14 Faghihzadeh F, Hekmatdoost A, Adibi P. Resveratrol and liver: A systematic review. J Res Med Sci. 2015 Aug;20(8):797-810.

15 Sener G, Toklu HZ, Sehirli AO, VelioğluOğünç A, Cetinel S, Gedik N. Protective ef- fects of resveratrol against acetaminopheninduced toxicity in mice. Hepatol Res. 2006 May;35(1):62-8.

16 Galal RM, Zaki HF, Seif El-Nasr MM, Agha AM. Potential protective effect of honey against paracetamol-induced hepatotoxicity. Arch Iran Med. 2012 Nov;15(11):67480.

17 Al Humayed S. Protective and therapeutic effects of Crataegus aronia in non-alcoholic fatty liver disease. Arch Physiol Biochem. 2017 Feb;123(1):23-30.

18 Al-Hashem F, Al-Humayed S, Amin SN, Kamar SS, Mansy SS, Hassan S, et al. Metformin inhibits mTOR-HIF- $1 \alpha$ axis and profibrogenic and inflammatory biomarkers in thioacetamide-induced hepatic tissue alterations.J Cell Physiol. 2019 Jun;234(6):9328-37.

19 Eleawa SM, Alkhateeb MA, Alhashem FH, Bin-Jaliah I, Sakr HF, Elrefaey HM, et al. Resveratrol reverses cadmium chloride-induced testicular damage and subfertility by downregulating p53 and Bax and upregulating gonadotropins and $\mathrm{Bcl}-2$ gene expression. J Reprod Dev. 2014 Apr;60(2):115-27.

20 Panasiuk A, Dzieciol J, Panasiuk B, Prokopowicz D. Expression of p53, Bax and Bcl-2 proteins in hepatocytes in non-alcoholic fatty liver disease. World J Gastroenterol. 2006 Oct;12(38):6198-202.

21 Williams CD, Bajt ML, Farhood A, Jaeschke $\mathrm{H}$. Acetaminophen-induced hepatic neutrophil accumulation and inflammatory liver injury in CD18-deficient mice. Liver Int. 2010 Oct;30(9):1280-92. 
22 Wang Y, Jiang Y, Fan X, Tan H, Zeng H, Wang $Y$, et al. Hepato-protective effect of resveratrol against acetaminophen-induced liver injury is associated with inhibition of CYPmediated bioactivation and regulation of SIRT1-p53 signaling pathways. Toxicol Lett. 2015 Jul;236(2):82-9.

23 Rivera H, Shibayama M, Tsutsumi V, PerezAlvarez V, Muriel P. Resveratrol and trimethylated resveratrol protect from acute liver damage induced by $\mathrm{CCl} 4$ in the rat. J Appl Toxicol. 2008 Mar;28(2):147-55.
24 El-Boghdady NA, Abdeltawab NF, Nooh MM. Resveratrol and Montelukast Alleviate Paraquat-Induced Hepatic Injury in Mice: Modulation of Oxidative Stress, Inflammation, and Apoptosis. Oxid Med Cell Longev. 2017;2017(10):9396425.

25 Boulares AH, Zoltoski AJ, Stoica BA, Cuvillier $\mathrm{O}$, Smulson ME. Acetaminophen induces a caspase-dependent and Bcl-XL sensitive apoptosis in human hepatoma cells and lymphocytes. Pharmacol Toxicol. 2002 Jan;90(1):38-50.

26 Pan S, Li S, Hu Y, Zhang H, Liu Y, Jiang H, et al. Resveratrol post-treatment protects against neonatal brain injury after hypoxia-ischemia. Oncotarget. 2016 Nov;7(48):79247-61.
27 Elbe H, Gul M, Cetin A, Taslidere E, Ozyalin F, Turkoz Y, et al. Resveratrol reduces light and electron microscopic changes in acetaminophen-induced hepatotoxicity in rats: role of iNOS expression. Ultrastruct Pathol. 2018 Jan-Feb;42(1):39-48.

28 Wang K. Molecular mechanisms of hepatic apoptosis. Cell Death Dis. 2014 Jan;5(5):e996.

29 Wojnarová L, Kutinová Canová N, Farghali $\mathrm{H}$, Kučera T. Sirtuin 1 modulation in rat model of acetaminophen-induced hepatotoxicity. Physiol Res. 2015;64(4 suppl 4):S477-87. 